\section{Pulmonary arterial aneurysm presenting as an endobronchial}

\section{mass}

\author{
P M Gibbs, A Hami
}

\begin{abstract}
The case is presented of a 25 year old man with haemoptysis who exsanguinated following endoscopic fine needle aspiration of an endobronchial mass. Necroscopic examination revealed an aneurysm of the proximal left pulmonary artery.

(Thorax 1995;50:1013-1014)
\end{abstract}

Keywords: aneurysm, pulmonary artery, bronchoscopy.

Pulmonary arterial aneurysms are rare and are usually associated with primary or secondary pulmonary hypertension, congenital heart anomalies, infection, collagen vascular diseases, or degenerative changes of the elastic media. ${ }^{1}$ Most cases are diagnosed radiographically or at necropsy, but a number of cases have been successfully treated by surgery. ${ }^{2}$ The majority of cases arise from the proximal pulmonary arteries and compress the surrounding parenchyma and vasculature. Although usually asymptomatic, they may cause dyspnoea, cough, haemoptysis, and chest pain. We report a case in which a small aneurysm eluded diagnosis via radiology and presented as an endobronchial mass.

\section{Case report}

A 25 year old Latin American man presented with a two day history of haemoptysis of one cupful of blood on two separate occasions. There was no history of fever, chills, chest pain, shortness of breath, or weight loss. He had insulin dependent diabetes mellitus, including episodes of diabetic ketoacidosis in the past, and a gunshot wound to the right mandible two years prior to admission. There was no history of bleeding diathesis, syphilis, tuberculosis, chest trauma, or intravenous drug use.

The patient's vital signs were normal and stable. Physical examination revealed no lesions of the eyes, ears, or oral cavity, but a small amount of oropharyngeal blood was seen. Decreased breath sounds were noted in the left lower lung field. Cardiac auscultation was normal. Fibreoptic endoscopic examination of the nasopharynx showed no evidence of epistaxis. Laboratory studies revealed a non-reactive serological test for syphilis, a normal coagulation screen, and a platelet count of $381 \times 10^{9} / 1$.

A chest radiograph demonstrated an infiltrate in the posterior segment of the left lower lobe of the lung. Fibreoptic bronchoscopy was performed, revealing a smooth, orange-red mass protruding into the posterior segment of the left lower lobe (fig 1). A fine needle aspiration biopsy was performed with a 21 gauge Stifcore needle (Microvasive, Watertown, Massachusetts, USA), resulting in brisk haemorrhage of approximately $1100 \mathrm{ml}$. The bleeding was uncontrolled by bronchoscopic tamponade or intrabronchial instillation of adrenaline. A Fogarty catheter was inserted into the left mainstem bronchus and inflated to provide tamponade. Temporary haemostasis was obtained, and the patient was transferred to the medical intensive care unit. Bronchial artery angiography was performed, revealing no evidence of vascular lesions or active bleeding. Repeat fibreoptic bronchoscopy was performed at the bedside on two subsequent occasions within 24 hours and showed no active bleeding. A lobectomy was planned, but was deferred to the next morning in the absence of demonstrable current haemorrhage. A sudden resumption of massive bleeding occurred late in the evening, and the patient died.

Necroscopic examination demonstrated a $2.0 \times 1.3 \mathrm{~cm}$ saccular aneurysm protruding into the bronchial lumen. The convex surface of the lesion consisted of a shell of partially organised fibrin, and bore a $1.1 \times 0.5 \mathrm{~cm}$ defect in direct communication with the lumen of the pulmonary artery (fig 2).

Histological examination showed fragmentation and dropout of elastic fibres in the aneurysmal wall, but no evidence of cystic medial necrosis. The portion of the aneurysmal wall protruding into the bronchus consisted of a fibrous neointima with scant collagen and no elastic fibres. Neither the lung nor any other tissue examined at necropsy showed histological evidence of granulomatous, luetic, or collagen vascular diseases.

\section{Discussion}

Though uncommon, pulmonary arterial aneurysms have been described in numerous case reviews and have been diagnosed both before and after death. ${ }^{12}$ An increasing number have been successfully treated with surgery in recent years; however, massive and often fatal haemoptysis reportedly occurs in $20-60 \%$ of cases, particularly with the solitary peripheral lesions. ${ }^{2}$ As most appear to rupture into the alveolar

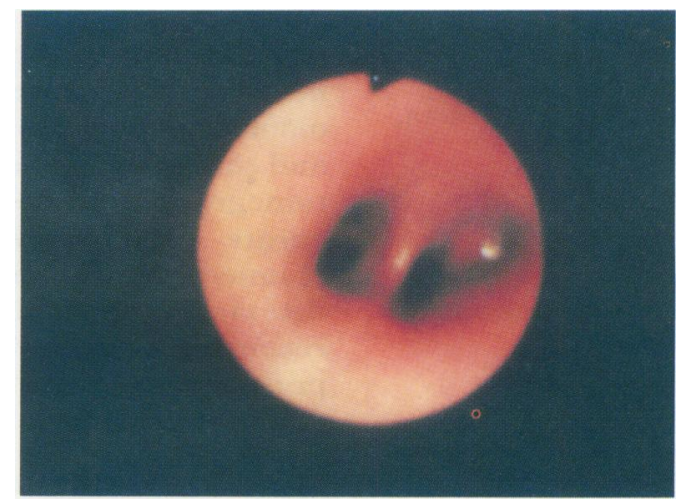

Figure 1 Bronchoscopic appearance of endobronchial pulmonary arterial aneurysm (left lower lobe, posterior bronchial segment). 


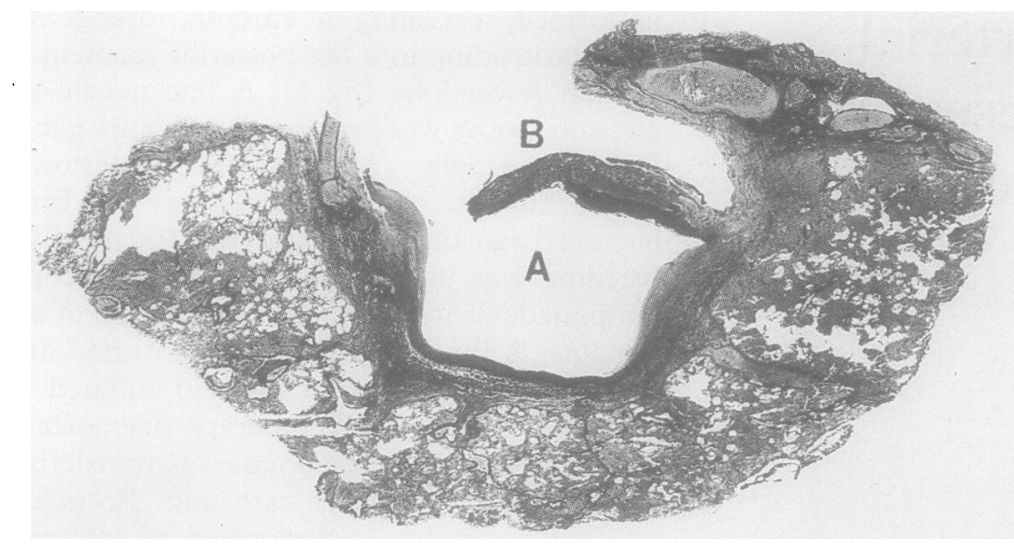

Figure 2 Paraffin embedded cross section of aneurysm at point of rupture into bronchial lumen; $A=$ aneurysmal lumen, $B=$ bronchial lumen. Elastic stain, magnification $\times 3$.

parenchyma and fail to impinge directly on a bronchus, it is not surprising that few have been detected by fibreoptic bronchoscopy.

We have found only one previous description of the bronchoscopic appearance of such a lesion. St Christov et $a l^{3}$ described a patient with a $2 \cdot 5-3 \mathrm{~cm}$ reddish, tumourlike mass filling the left main bronchus. This lesion bled spontaneously after a few seconds and resulted in the patient's death. Pathological examination revealed a bulbous aneurysm encasing the bronchus in a cuff-like fashion, with medial necrosis of the vessel wall. Erosion into the bronchi must almost certainly occur in many proximal aneurysms, given the frequency of fatal haemoptysis, but we found no other report of bronchoscopic detection of an aneurysm.

The current case is notable for its mimicry of a submucosal tumour. The bland, orangered bronchoscopic appearance belied the vascular nature of the lesion, unlike the more telltale, plexiform red pattern seen in well vascularised tumours such as a central carcinoid. Although transection of normal pulmonary vessels has been reported as a complication of transbronchial biopsy, ${ }^{4}$ such vessels are unlikely to protrude into the airway lumen.

Previous reports ${ }^{5}$ have cautioned against percutaneous biopsy of peripheral coin lesions without prior radiological examination, such as rapid sequence $\mathrm{CT}$ scan with contrast, to rule out aneurysms. This case suggests that similar caution needs to be extended to selected bronchoscopic procedures, and that the bronchoscopist would do well to add this lesion to the list of endobronchial masses in which a biopsy is to be assiduously avoided.

\section{Butto F, Lucas RV, Edwards JE. Pulmonary arterial aneurysm. A pathologic study of five cases. Chest 1987;91:237-41. \\ 2 Ungaro R, Saab S, Almond CH, Kumar S. Solitary periphera pulmonary artery aneurysms. Pathogenesis and surgica treatment. F Thorac Cardiovasc Surg 1976;71:566-71. \\ 3 St Christov V, Kanasirski P. Aneurysma des linken Haupastes der Arteria pulmonaris mit Ruptur in den linken Hauptbronchus. Zentralbl Alg Pathol 1966;109:524-6. \\ 4 Herf SM, Suratt PM. Complications of transbronchial lung biopsies. Chest 1978;Suppl 5:759-60. \\ 5 Eisenberg D, Gordon RL, Weineman EE, Romanoff $H$. Pulmonary artery aneurysm presenting as a peripheral coin lesion - the danger of needle biopsy. Cardiovasc Intervent Radiol 1984;7:280-2.}

\section{Preoperative diagnosis of a pulmonary artery} sarcoma

\section{Cardiovascular Surgery Unit $\checkmark$ Velebit $\mathrm{J}$ T Christenson \\ F Simonet \\ J Maurice \\ M Schmuziger}

\section{Department of Radiology} $\mathrm{H}$ Hauser

Hôpital de la Tour, 1217 Meyrin-Geneva, Switzerland

\section{Department of} Radiology, Hôpital Cantonal Universitaire, Geneva, Switzerland D Didier

Reprint requests to: Dr V Velebit.

Received 16 May 1994 Returned to authors 5 September 1994 Revised version received 6 October 1994 Accepted for publication 18 October 1994

\author{
V Velebit, J T Christenson, F Simonet, \\ J Maurice, M Schmuziger, H Hauser, \\ D Didier
}

\begin{abstract}
A pulmonary artery sarcoma was diagnosed preoperatively by magnetic resonance imaging enhanced with gadolinium and confirmed by percutaneous computed tomographic guided needle biopsy. Accurate preoperative diagnosis allowed planned curative surgery with removal of the right ventricular outflow tract and reconstructive surgery using a cryopreserved homograft.
\end{abstract}

(Thorax 1995;50:1014-1015)

Keywords: pulmonary artery sarcoma, diagnosis.

Pulmonary artery sarcoma is a rare tumour of the cardiovascular system, only about 120 cases having been reported. ${ }^{1}$ The tumour is most often mistaken for pulmonary embolism, ${ }^{2}$ and the surgical treatment may have to be improvised if the diagnosis is established only upon exploration of the pulmonary artery, which may lead to incomplete resection, palliative surgery, or imperfect reconstruction. ${ }^{3}$ The advantage of a precise preoperative diagnosis is obvious. We report a case of pulmonary artery sarcoma which was diagnosed preoperatively using two readily available radiological techniques that allowed planned and appropriate surgery.

\section{Case report}

A 37 year old man was admitted for suspected pulmonary embolism. He had voluntarily lost $7 \mathrm{~kg}$ in weight over the previous three months and presented with a dry cough and intermittent fleeting pain in the left chest. A chest radiograph showed reduced vascularisation of the left lung and a ventilation perfusion scan showed absence of perfusion to the left lung. The patient was not in distress and physical examination and blood gas analysis breathing air were normal. An ascending venogram showed no thrombi in the leg veins and vena cava. The pulmonary artery pressure was normal. A computed tomographic (CT) scan of the thorax confirmed the presence of an obstructed 